\title{
Malignant fibrous histiocytoma of the deep peri-articular tissue of the stifle in a dog
}

\author{
M J Booth ${ }^{a}$, S S Bastianello ${ }^{c}, M_{\text {Jiminez }}{ }^{a, b}$, A van Heerden $^{d}$
}

\begin{abstract}
A Belgian shepherd dog aged 4 years and 9 months was presented with acute onset of non-weight bearing lameness and stifle effusion of the left hind limb, caused by the deep form of a malignant fibrous histiocytoma originating in the deep musculature and fascia surrounding the stifle joint. The tumour progressed rapidly in the tissues along the femoral diaphysis with marked periosteal new bone formation. Cytology of a stifle joint aspirate revealed numerous large polygonal neoplastic cells with considerable anisocytosis and anisokaryosis. These cells were present in clusters, with cytoplasmic projections between the cells, but occasionally also occurred singly. Several cells contained multiple cytoplasmic vacuoles and occasional giant cells were also encountered. Adequate tumour-free margins were not possible with radical limb amputation and the dog was euthanased. Macroscopically the tumour appeared as an extensive pale tan, firm mass with scattered small haemorrhages and foci of yellow discolouration. Histologically the tumour consisted of dense neoplastic expanses or multiple nodules, composed of spindle-shaped fibroblastic cells, polygonal histiocytic cells or cell clusters and scattered giant cells with 2-3 nuclei. The polygonal neoplastic cells were frequently present around and invading lymphatics and blood vessels, causing neoplastic emboli. This is the 1st report of the clinical behaviour, radiography and cytology of the deep form of malignant fibrous histiocytoma in the dog.
\end{abstract}

Key words: cytology, dog, malignant fibrous histiocytoma, malignant giant cell tumour of soft parts, radiology.

Booth M J, Bastianello S S, Jiminez M, Van Heerden A Malignant fibrous histiocytoma of the deep peri-articular tissue of the stifle in a dog. Journal of the South African Veterinary Association (1998) 69(4): 163-168 (En.). Department of Surgery, Faculty of Veterinary Science, University of Pretoria, Private Bag X04, Onderstepoort, 0110 South Africa.

\section{INTRODUCTION}

The incidence of tumours of periarticular soft tissues, namely tendon sheaths, bursae and fascia in domestic animals has not been reported. Of 2361 canine neoplasms in the United Kingdom, all skeletal tumours, including those of bone, tendon sheath and muscle origin, comprised $4.1 \%$, and non-bone skeletal tumours $0.3 \%$ of all canine tumours ${ }^{4}$. In a review of 30 canine joint tumours, only $27 \%$ were synovial sarcomas ${ }^{19}$, contrary to the previously accepted notion that most joint tumours are synovial sarco$\operatorname{mas}^{13}$.

Malignant fibrous histiocytoma (malignant giant-cell tumour of soft parts) is a

aDepartment of Surgery, Faculty of Veterinary Science, University of Pretoria, Private Bag X04, Onderstepoort, 0110 South Africa.

${ }^{\mathrm{b}}$ Deceased.

${ }^{\mathrm{C} P a t h o l o g y}$ Section, Onderstepoort Veterinary Research Institute, Private Bag X05, Onderstepoort, 0110 South Africa.

dDepartment of Medicine, Faculty of Veterinary Science, University of Pretoria, Private Bag X04, Onderstepoort, 0110 South Africa.

Received: April 1998. Accepted: October 1998. rare extraskeletal neoplasm of the superficial and deep connective tissues, occurring in humans and animals. In the veterinary literature it has been reported in a mule ${ }^{5}, 7$ horses $^{6,14}, 12$ cats $^{1-3,6-9,12,1,1,17}$, $3 \operatorname{dogs}^{13}$ and 3 Syrian golden hamsters ${ }^{16}$. The 3 dog cases formed part of a pathology collection with no published clinical reports of the condition. Early reports proposed that the deep form of this tumour arose from tendons or tendon sheaths, resulting in the term 'malignant giant-cell tumour of tendon sheaths ${ }^{12}$. Ultrastructural and tissue culture studies suggest that their origin is from pluripotential undifferentiated mesenchymal cells $^{3,6,11,14}$. A variety of synonyms have been used to describe the deep variant of this neoplasm, namely extraskeletal giant-cell tumour, malignant giant-cell tumour of tendon sheaths, giant-cell variant of synovial sarcoma of tendons and tendon sheaths, and malignant fibrous histiocytoma. A review article proposed that all human malignant soft tissue tumours with a common fibroushistiocytic cell type origin should be reclassified as malignant fibrous histiocytomas ${ }^{11}$. In the veterinary literature this tumour is commonly referred to as malignant fibrous histiocytoma ${ }^{2,3,7-9,15,17}$ or malignant giant-cell tumour of soft parts $^{6,13,14}$.

Superficial and deep forms of this tumour have been recognised based on location and clinical behaviour ${ }^{10,11,13}$. In man the superficial form comprises small tumours, occurring in the subcutis and superficial fascia of the leg in particular, that rarely metastasise but are prone to local recurrence ${ }^{10,11}$. The deep tumours are large aggressive neoplasms with a predilection for skeletal muscle, deep fascia and tendons of particularly the thigh, and frequently metastasise $e^{10,11}$. The tumours in equines have all been of the superficial type with a predilection for the hind $\operatorname{limb}^{5,6,13,14}$. In cats and dogs, deep $^{1,6,9,12,13,15,17}$ and superficial forms ${ }^{3,7-9,13,19}$ have been documented. Local recurrence has been reported in $4 / 4$ cats with superficial and deep types where complete surgical excision was accomplished and cases followed-up ${ }^{3,6,9}$.

There are apparently no published clinical reports of the deep variant of a malignant fibrous histiocytoma in a dog. The purpose of this article is to report the clinical, radiographic, cytological and histopathological findings of such a case.

\section{CASE DESCRIPTION}

\section{History and clinical presentation}

A spayed female Belgian shepherd dog aged 4 year and 9 months, body weight $22 \mathrm{~kg}$, was referred to the Onderstepoort Veterinary Academic Hospital (OVAH) with hind-limb lameness of 15 days' duration. The initial complaint was acute onset grade $2 / 4$ lameness with pain in the left hind limb. A radiograph (Fig. 1) made by the referring veterinarian 10 days earlier showed a 2-mm thick, palisade to solid periosteal reaction on the cranial femoral cortex from the proximal limit of the trochlea to the mid-diaphyseal region. A similar periosteal reaction was present caudally for $2 \mathrm{~cm}$ just proximal to the gastrocnemius muscle insertion. Early permeative lysis was seen in the distal 


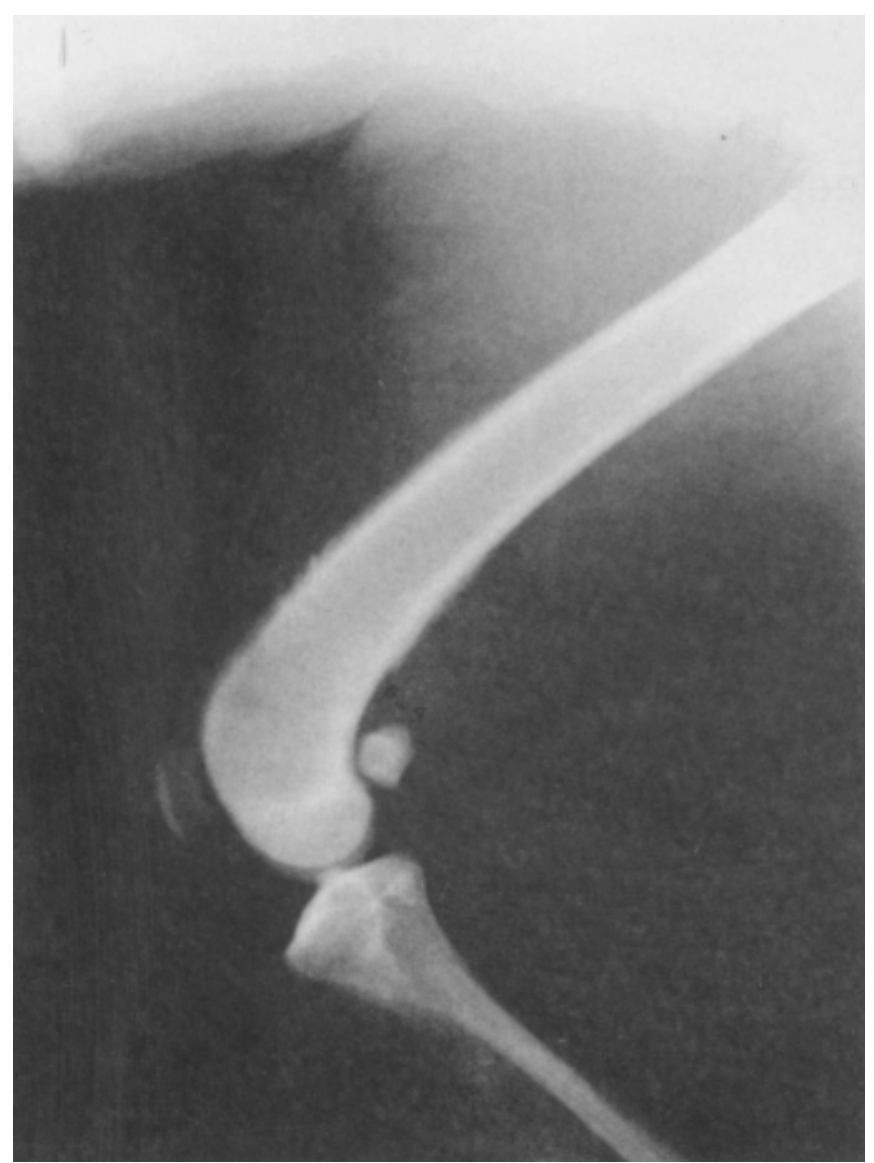

Fig. 1: Radiograph made 10 days before referral to OVAH, showing periosteal reactions and early permeative lysis.

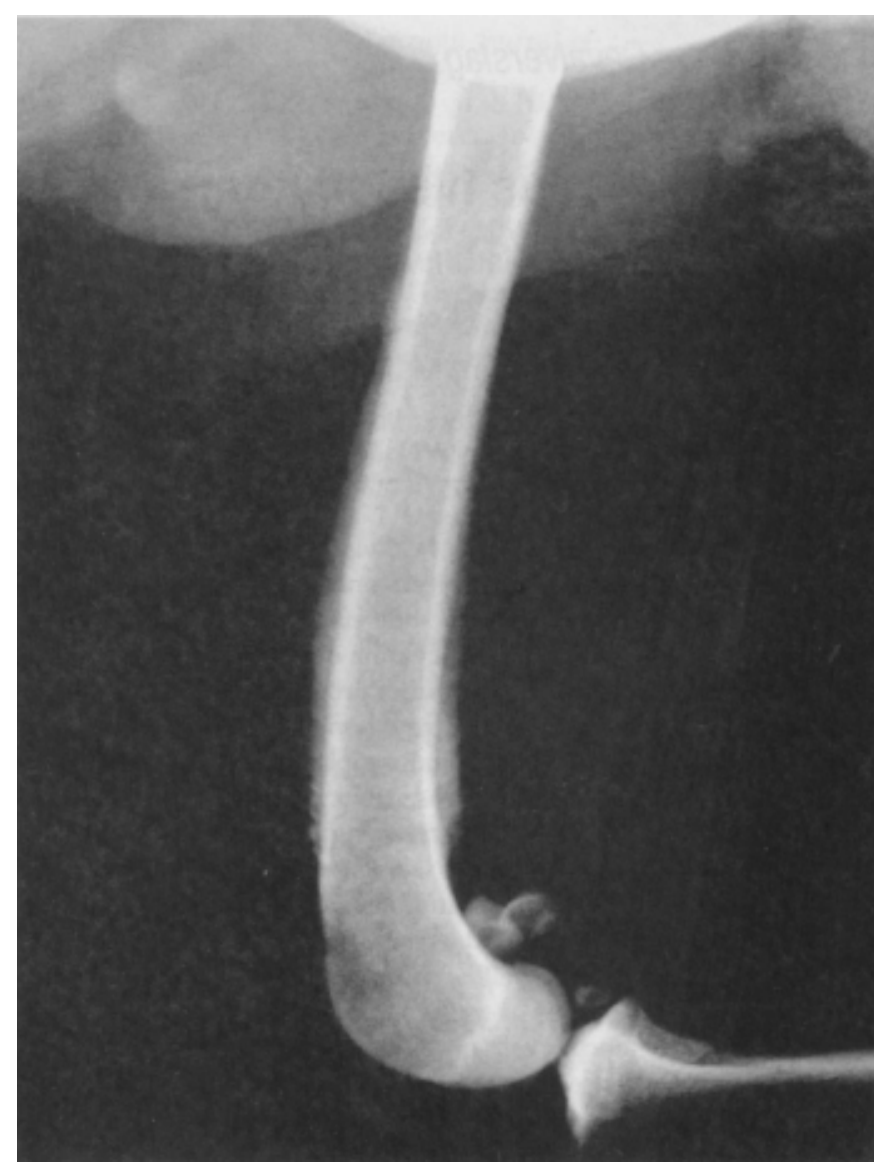

Fig. 2: Radiograph made on arrival at OVAH, showing progression of the periosteal reactions. femoral diaphysis. Enrofloxacin (Baytril, Bayer) and phenylbutazone (Butrex, Noristan Laboratories) had been prescribed, but the lameness had progressively worsened. Physical examination at OVAH revealed an animal in good condition with a non-weight-bearing left hind-limb lameness. A diffuse, firm, painful swelling of the stifle and distal femoral region was present. Examination of a peripheral blood smear, full urinalysis and faecal flotation were normal. Routine haematology was within normal reference ranges.

Radiography (Fig. 2) showed that the periosteal reaction had thickened to $5 \mathrm{~mm}$ and extended to involve the entire diaphysis. Mild subperiosteal scalloping was present with slight progression of the permeative lysis. A poorly-defined soft-tissue density $6 \times 2 \mathrm{~cm}$ was located adjacent to the caudodistal femoral shaft and stifle effusion was present.

A standard lateral parapatellar arthrotomy of the left stifle revealed a severe joint effusion. Samples of this haemorrhagic fluid were aspirated before incision into the joint capsule, and submitted for cytological and microbiological examination. The joint capsule was markedly thickened to about $10 \mathrm{~mm}$ but smooth, with no other macroscopic lesions evident. The intra-capsular distal femur exhibited a diffuse tissue reaction with the exception of the articular cartilage. This tissue was firm, friable, red-orange in colour with a granular surface resembling granulation tissue, well demarcated and raised 1-2 $\mathrm{mm}$ above the surface of the bone. Bone from the lateral femoral epicondyle was biopsied using a Michelle's trephine, and submitted together with the soft tissue reaction in $10 \%$ buffered formalin for histopathological examination. Bone marrow was obtained from the distal femur for microbiological examination. The joint was copiously lavaged with lactated Ringers (Ringer Lactate, Intramed) and routinely closed after placement of a penrose drain to allow drainage from the joint. A sterile dressing was applied and the dog placed on amoxycillin-clavulanic acid (Synulox, Smith-Kline Beecham) and metronidazole (Flagyl, Maybaker) twice daily per os awaiting the culture results.

On cytological examination of the joint aspirate, high cellularity and a large quantity of myxoid material were noted. White blood cell numbers were high, comprising mainly mature neutrophils and very active macrophages, but no microorganisms were seen. Neoplastic cells were present in large numbers, usually in clusters but occasionally singly (Fig. 3). The neoplastic cells were large, with a fairly clear cytoplasmic membrane with cytoplasmic connections evident between some cells. Marked anisocytosis and anisokaryosis were evident. The cytoplasm was markedly basophilic, moderate in amount, and several cells contained multiple cytoplasmic vacuoles (Fig. 3). The nuclei were large, rounded, and eccentrically positioned with large, irregular nucleoli. Binucleated and occasional multi-nucleated forms were seen. A provisional diagnosis of chondrosarcoma or osteosarcoma was made based on the cytological findings.

Aerobic culture of the joint fluid aspirate yielded only 3 colonies of a coagulase-positive Staphylococcus sp. Anaerobic and fungal cultures yielded no growth, and no cultures were obtained from the bone-marrow sample. A diagnosis of synovial sarcoma was made on the biopsy samples by the duty pathologist.

Thoracic radiographs revealed no evidence of pulmonary metastasis and an abdominal ultrasound examination was within normal limits. Follow-up radiographs (Fig. 4) made 6 days later showed progression of the periosteal reactions, which had become thicker and more aggressive, evidenced by a greater 


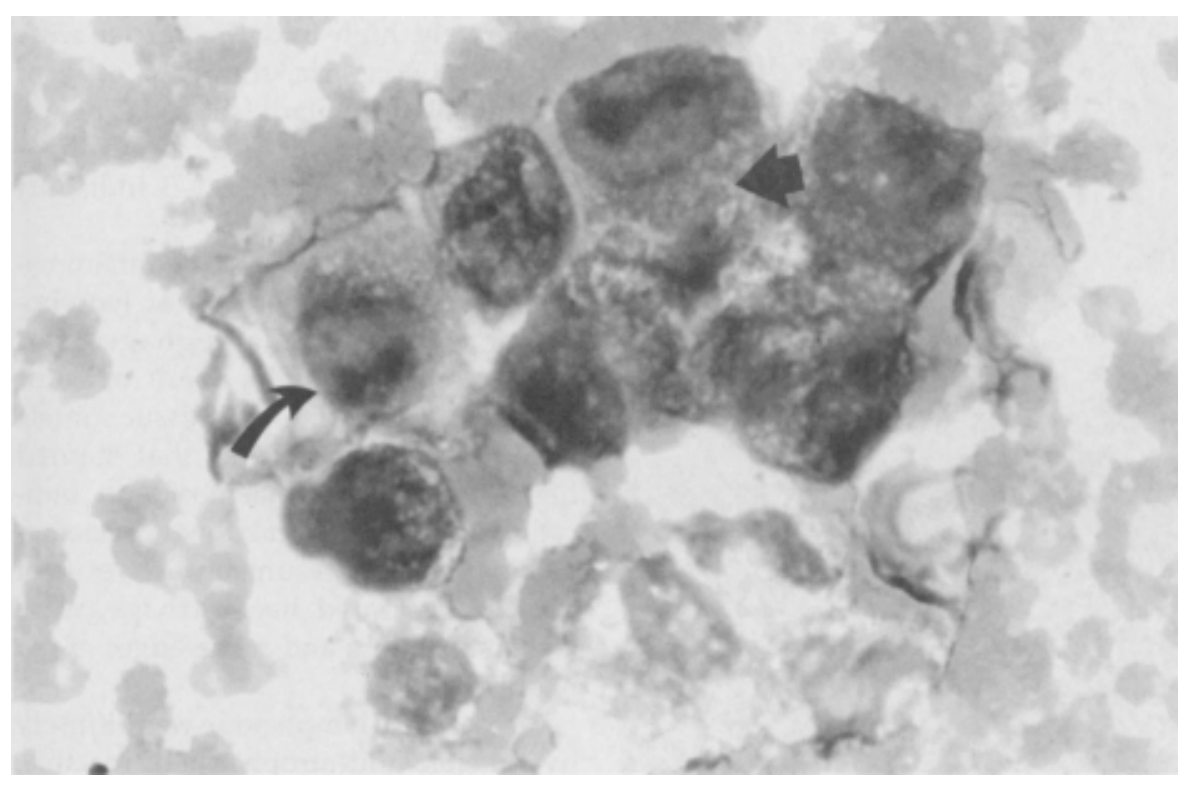

Fig. 3: Cytology, joint aspirate: cluster of polygonal neoplastic cells. Note dark eccentric nuclei (curved arrow) and moderately vacuolated cytoplasm (broad arrow) Romanowsky stain, $\times 1200)$.

sunburst reaction proximally. The lesion was regarded as neoplastic in type and originating in the deep periosseous soft tissue rather than the bone.

The tumour was regarded as aggressive in nature based on the rapid progression of the radiological changes. A guarded prognosis was given and hind-limb amputation by coxofemoral disarticulation and resection of the thigh muscles advised. During surgery the superficial thigh muscles appeared firm and pale.

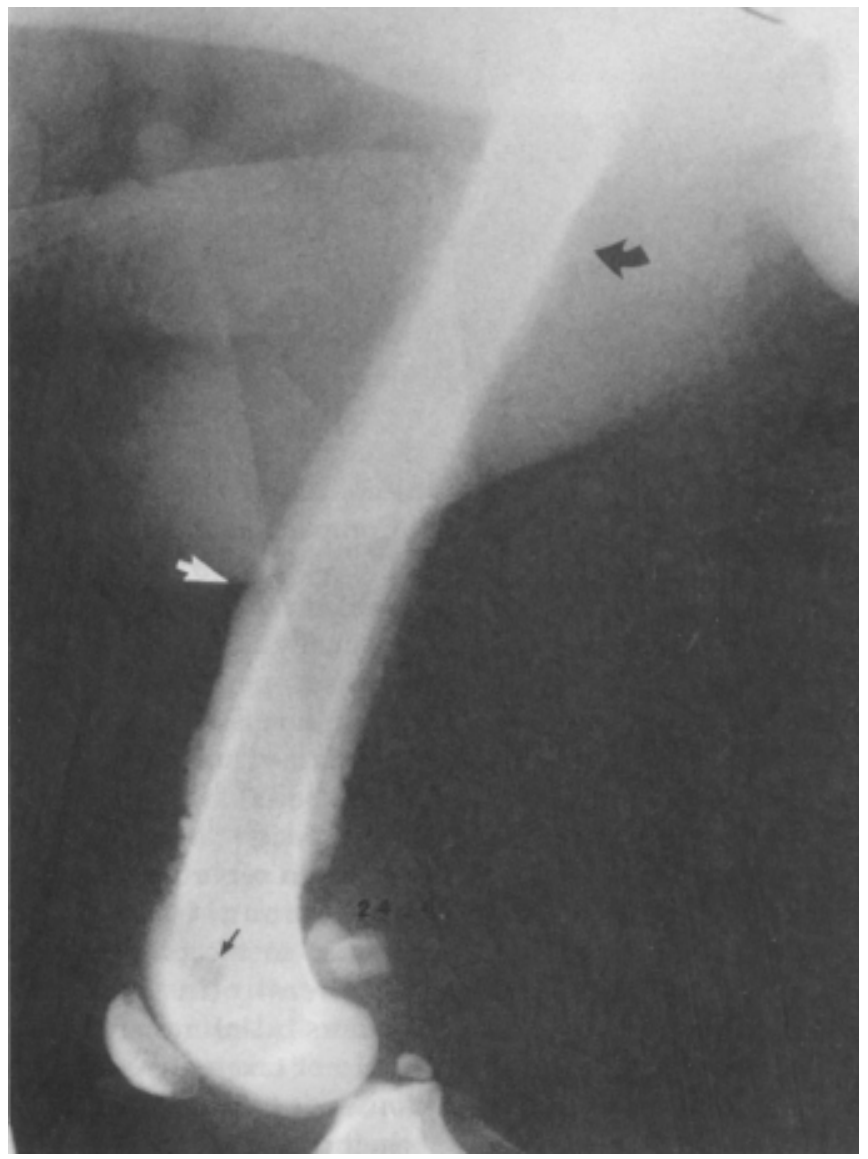

Fig. 4: Radiograph made 6 days after presentation to OVAH, showing a thick palisade periosteal reaction (white arrow), becoming more sunburst proximally (large black arrow). The area of the bone biopsy is indicated by the small black arrow distally.

The muscles surrounding the coxofemoral joint had a similar appearance and a small amount of haemorrhagic fluid was present within the joint. Owing to the poor prognosis and inability to obtain adequate tumour-free margins via the amputation, euthanasia was performed under anaesthesia.

\section{Pathological findings}

At necropsy a large pale tan, firm mass with scattered small haemorrhages and yellowish areas was present in the deeper periosseous skeletal muscles and fascia of the distal thigh, surrounding the stifle joint but not involving the proximal tibia. The skeletal muscle groups not invaded or replaced by neoplastic tissue were poorly delineated, pale and oedematous in appearance. There was no clear margin between normal and diseased tissue. The intra-articular appearance of the stifle was the same as seen during arthrotomy, without effusion, as the penrose drain was still in position. The osseous and medullary tissue of the femur were normal in appearance. A 5-6-mm thick, irregular to solid periosteal reaction completely surrounded the femoral diaphysis. The popliteal lymph node was haemorrhagic but normal in size. The skeletal muscles of the proximal thigh appeared pale but there were no haemorrhages and the individual muscles were distinct. No macroscopic pulmonary pathology was present.

Samples of diseased tissue surrounding the affected coxofemoral joint, femoral shaft and popliteal lymph node were collected in $10 \%$ buffered formalin. The tissues were routinely processed and stained with haemotoxylin and eosin (HE), Prussian blue and Schmorls' stains for haemosiderin and lipofuscin pigment respectively.

The diseased periosseous tissue comprised multiple neoplastic expanses or nodules surrounded by mature collagenous or active fibroblastic, highly vascularised granulation tissue. Remnants of pre-existing loose connective tissue and adipose tissue were also noted. The neoplastic foci were present as dense sheets of cells (Fig. 5) or single to multinodular cellular aggregates in close association with one or more lymphatics or blood vessels (Figs 6, 7). Neoplastic cells could be seen actively invading the walls of several of these blood vessels and most of the lumens were partially or completely occluded by neoplastic emboli (Figs 6, 7), the larger of which revealed central necrosis.

The neoplastic tissue consisted of a mixture of predominantly dense fibrosarcomatous areas of elongated, spindle- 


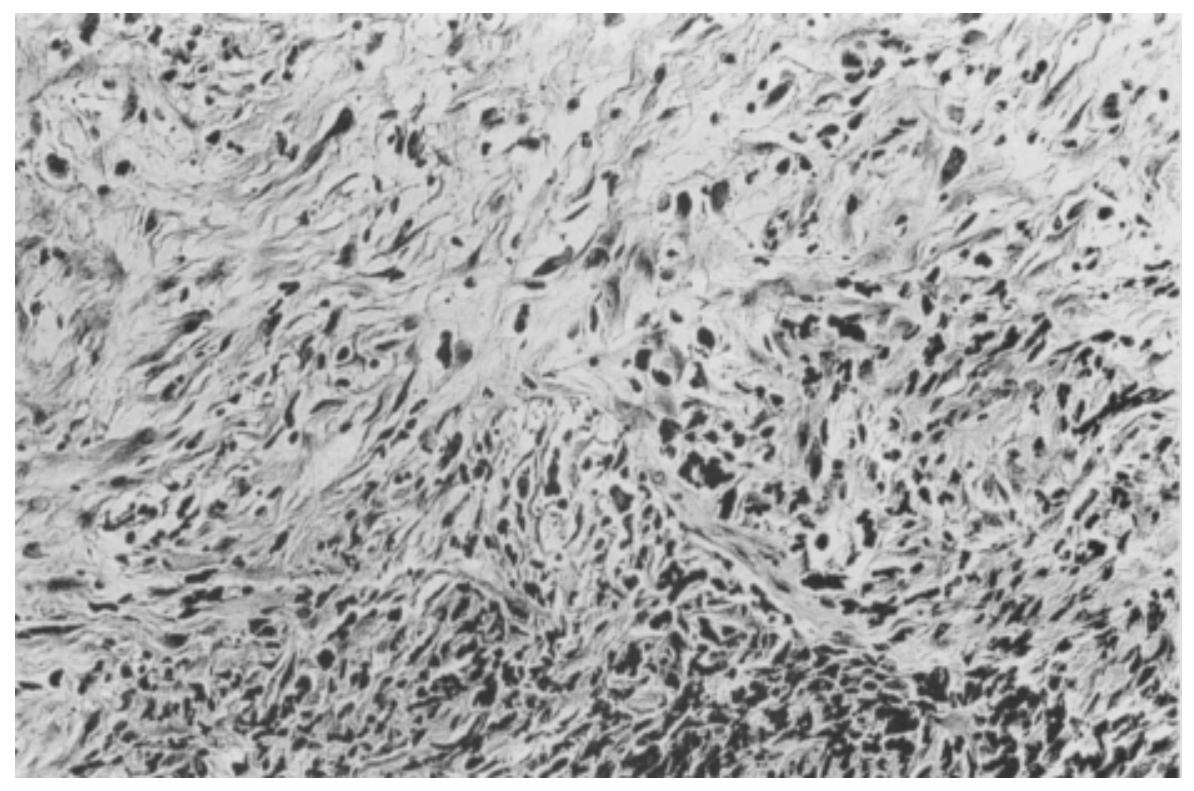

Fig. 5: Primary tumour, fibromatous appearance. The tissue consists predominantly of elongated spindle-shaped cells with scattered histiocytic cells (HE, $\times 120)$.

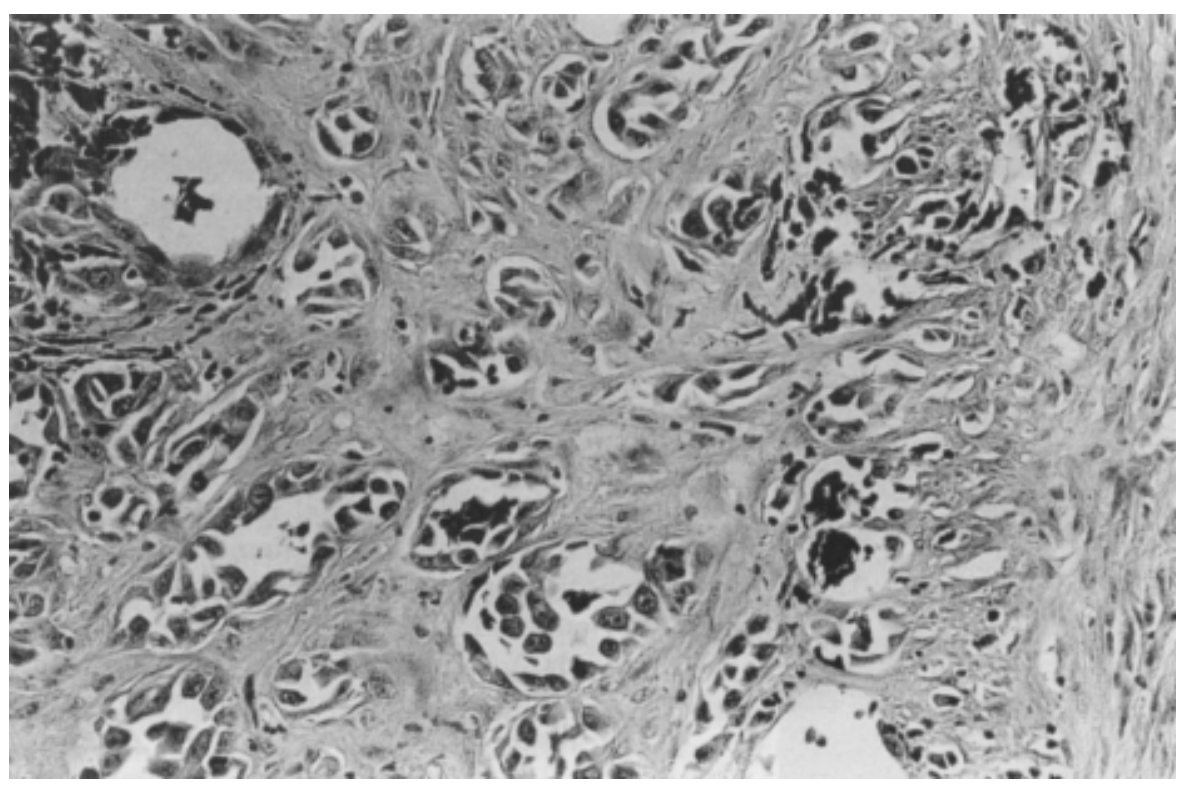

Fig. 6: Primary tumour: numerous lymphatics-containing tumour emboli embedded within neoplastic tissue. The emboli partially occlude the vessel lumens and frequently occur as a layer lining the vessel wall $(\mathrm{HE}, \times 320)$.

shaped cells (Fig. 5) and more cellular areas consisting of rounded, highly pleomorphic histiocytic-type cells and plump, spindle-shaped cells (Figs 5,8). The neoplastic cells comprising the emboli were generally of the rounded polygonal type (Figs 6, 7). The areas with polygonal or plump, spindle-shaped cells revealed moderate pleomorphism and anaplasia but a relatively low mitotic index, averaging 1.5 per high power field. The spindle-shaped cells had indistinct eosinophilic cytoplasm and central nuclei while the polygonal cells had a moderate amount of eosinophilic to amphophilic cytoplasm. Several of the polygonal cells contained golden-brown pigment granules that stained positively with Prussian blue stain, or multiple small vacuoles, suggestive of phagocytic vacuoles, indicating that these were probably of histiocytic or macrophage origin. Binucleated or multinucleated giant cells or clusters of 2-3 closely apposed polygonal cells (Fig. 8) were scattered throughout the tumour, particularly in the histiocytic areas. The nuclear:cytoplasmic ratio of the polygonal cells was moderately high and the nuclei were central or eccentric, round to oval, or occasionally irregularly shaped. The nuclei stained normo- or hyperchromatically and contained 1-3 indistinct nucleoli.

Haemorrhages and foci of inflammatory round cells consisting of lymphocytes, pigmented macrophages and isolated plasma cells were present throughout the tumorous tissue. Single pigmented macrophages that stained highly positively for haemosiderin, indicating uptake and breakdown of haemoglobin pigment, presumably as a result of tumour-associated haemorrhage, were dispersed within and at the edges of the neoplastic foci.

The popliteal lymph node was diffusely hyperaemic and atrophic with inactive, depleted cortical lymphoid nodules and hypocellular medullary cords. A single neoplastic focus was evident in the cortex immediately below the capsule. The subcapsular and intermediate sinuses in this region contained neoplastic emboli, the cells of which were of the anaplastic polygonal type as described for the primary tumour (Fig. 9). A fresh haemorrhage occurred in the hilar region and the sinuses contained scattered erythrocytes. Haemosiderin pigment granules (Prussian-blue positive) were present in the cells of the mononuclear-phagocytic system.

\section{DISCUSSION}

The tumour described in this report grew rapidly within 2-3 weeks of clinical signs being noticed, involved an extensive area of the deep thigh musculature and fascia and had metastasised to the regional popliteal lymph node, indicative of the deep form of a malignant fibrous histiocytoma ${ }^{10,11,13}$.

Comparison of radiographs made on admission to OVAH and 6 days later revealed rapid progression of the lesion proximally along the femur, evidenced by increased periosteal thickening. Rapid progression is a prominent feature of the deep form of malignant giant-cell tumour of soft parts ${ }^{10,11,13}$. A periosteal reaction ${ }^{15}$, cortical lysis $^{9,15,17}$ and metaplastic bone ${ }^{9,13,17}$ have been reported in the deep form of this tumour. Osteoid was present adjacent to the connective tissue septa peripherally in $11 / 20$ deep human tumours $^{10}$. Unfortunately bone samples were not taken at post mortem examination, so it was not possible to determine whether the new bone formation was caused by periosteal elevation, metaplasia or neoplasia in this dog.

These tumours are reported to be non-painful $1^{10,12}$, causing lameness of a 


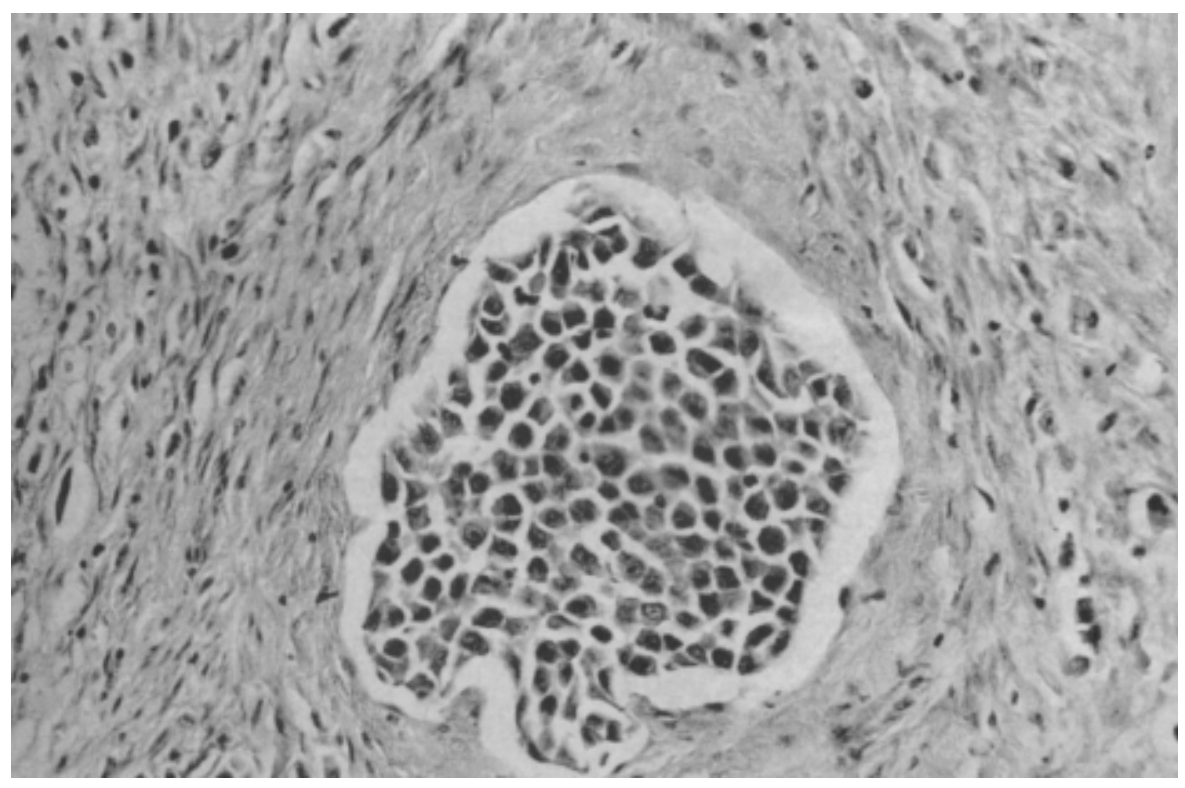

Fig. 7: Primary tumour: a lymph vessel embedded within dense tumorous tissue containing a large neoplastic embolus occluding the lumen $(\mathrm{HE}, \times 350)$.

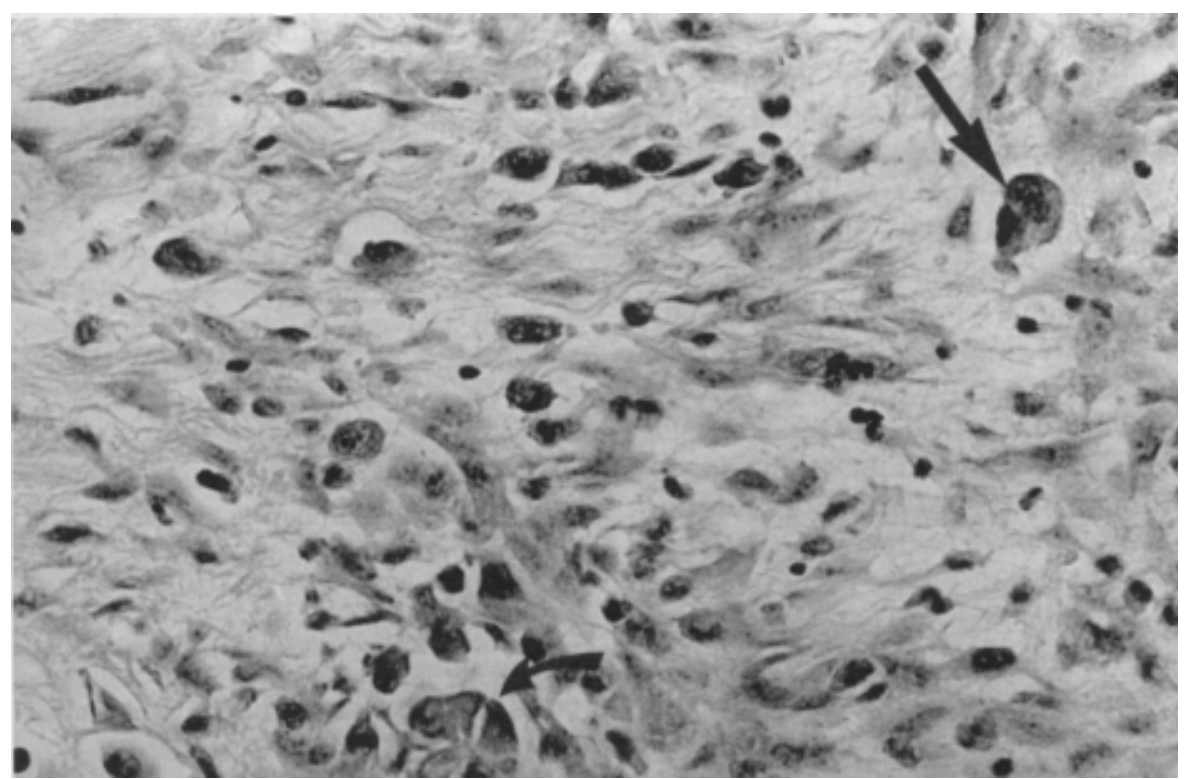

Fig. 8: Primary tumour: pleomorphic histiocytic neoplastic cells within a collagenous background matrix. Note the multinucleate cell (straight arrow) and cluster of neoplastic cells (curved arrow) (HE, $\times 400)$.

mechanical nature ${ }^{1,2,8,13}$. The pain present in this case is thought to have resulted from severe joint distension of the stifle caused by the neoplastic effusion from the intra-articular tumour. In a previous case of a deep malignant fibrous histiocytoma involving the metatarsophalangeal joint of a cat, no joint effusion was noted, and the articular surfaces were also unaffected ${ }^{15}$. Bacterial culture excluded an infectious aetiology. Isolation of 3 bacterial colonies was believed to be due to cutaneous contaminants based on their low numbers, the absence of bacteria on cytological examination and the lack of bacterial growth from granulation tissue (both of which are suggestive of synovial sarcoma) ${ }^{13}$, with no evidence of histiocytes or giant cells.

The actual diagnosis of the type of tumour involved was only made upon histopathological examination of the neoplastic tissue obtained at necropsy. The latter tissue presented 2 features of diagnostic significance: it was composed of a mixture of 3 cell types, namely elongated or plump spindle-shaped cells, rounded or polygonal cells resembling histiocytes or macrophages, and scattered giant cells; and the neoplastic cells were arranged in a multinodular fashion around lymphatics and blood vessels. These features are consistent with those described for malignant giant-cell tumour of soft parts ${ }^{1,3,6,10,13,14,16}$. There is much controversy as to the cell of origin of this tumour, but the present consensus of opinion is that it arises from pluripotential undifferentiated mesenchymal cells, which then differentiate to fibroblasts, histiocytes or intermediate cell types ${ }^{3,11,13}$. The giant cells probably arise by incomplete cell division i.e. nuclear division without concomitant cytoplasmic division, which may be a feature of several rapidly-growing malignant neoplasms ${ }^{1}$. In a series of 32 cases of malignant fibrous histiocytoma in humans, Guccion \& Enzinger ${ }^{10}$ noted vacuoles in several neoplastic histiocytes and giant cells as well as multifocal haemorrhages and haemosiderin accumulation in histiocytes adjacent to the haemorrhagic areas. All of these features were observed in the present case.

Cytological examination of fine-needle aspirates ${ }^{1}$ and tissue-fluid aspirates ${ }^{8}$ from malignant fibrous histiocytoma have been reported in 2 cats, but this is the 1st time that the cytology of the joint effusion from such a tumour has been described. Of interest in the present case was the good correlation between histopathological and cytological examinations with regard to the occurrence and appearance of the neoplastic cells of the polygonal and giant-cell forms. Although 'fibroblastoid' cells were seen on cytology in one report ${ }^{8}$, the fact that spindle-shaped neoplastic cells were not observed in our case can be explained by the nature of this tumour, as these cells would be more firmly embedded in the solid neoplastic tissue than histiocytes and giant cells and would therefore probably not appear in a preparation of fluid derived from a neoplastic focus. The tumour described in this report had extensive fibrosarcomatous areas, which is a feature of malignancy in this tumour type ${ }^{13}$. The occurrence of cell clusters and the presence of cytoplasmic connections 


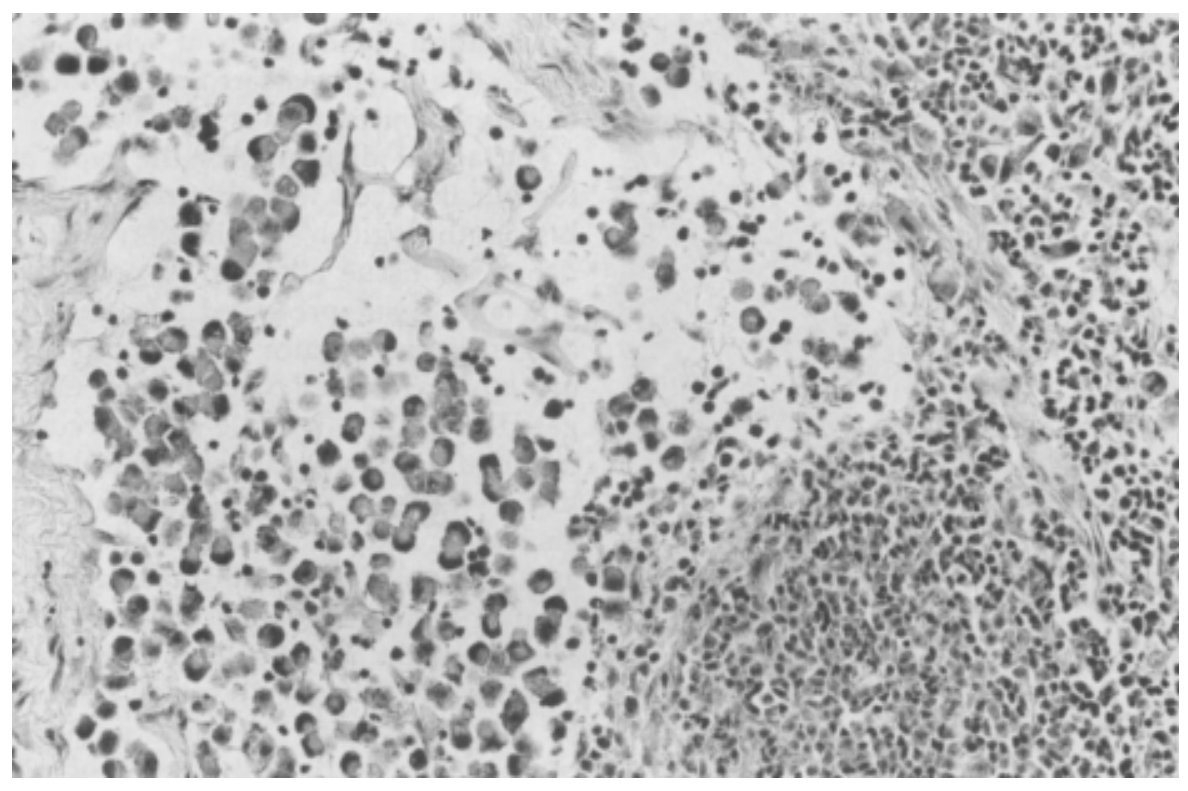

Fig. 9: Metastatic focus, popliteal lymph node: polygonal neoplastic cells in a cortical sinus. Normal lymph node tissue is evident on the right-hand side $(\mathrm{HE}, \times 320)$.

between some of the cells on cytology is consistent with the ultrastructural findings of the presence of cytoplasmic projections with histiocytes in malignant fibrous histiocytomas ${ }^{3}$.

Lattes ${ }^{11}$ noted that malignant giant cell tumours of soft parts, unlike fibrosarcomas, showed a tendency to metastasise via both the lymphatic and haematogenous routes with consequent involvement of regional lymph nodes. Regional lymph-node metastasis has been reported in a cat ${ }^{8}$ and $a \operatorname{dog}^{13}$. A metastatic focus was present in the regional popliteal lymph node in this dog, supporting this observation. Of interest in the tumour reported here was the presence of neoplastic polygonal and giant cells clustered around and invading lymphatics and blood vessels with consequent development of numerous emboli. This feature has been described as a common occurrence in the deep form of malignant histiocytomas in humans ${ }^{11}$, but except for reports of 6 cases in horses and 3 in Syrian golden hamsters ${ }^{14,16}$, does not
3. Confer A W, Enright F M, Beard G B 1981 Ultrastructure of a feline extraskeletal giant cell tumor (malignant fibrous histiocytoma). Veterinary Pathology 18: 738-744

4. Cotchin E 1954 Neoplasia in the dog. Veterinary Record 66: 879-888

5. Danks A G, Olafson P 1939 Giant cell sarcoma. Cornell Veterinarian 29: 68-70

6. Ford G H, Empson R N, Plopper C G, Brown P H 1975 Giant cell tumor of soft parts: a report of an equine and a feline case. Veterinary Pathology 12: 428-433

7. Garma-Aviña A 1987 Malignant fibrous histiocytoma of the giant cell type in a cat. Journal of Comparative Pathology 97: 551-557

8. Gibson K L, Blass C E, Simpson M, Gaunt S D 1989 Malignant fibrous histiocytoma in a cat. Journal of the American Veterinary Medical Association 194: 1443-1445

9. Gleiser C A, Raulston G L, Jardine J H, Gray KN 1979 Malignant fibrous histiocytoma in dogs and cats. Veterinary Pathology 16: 199-208

10. Guccion J G, Enzinger F M 1972 Malignant giant cell tumor of soft parts: an analysis of 32 cases. Cancer 29: 1518-1529

11. Lattes R 1982 Malignant fibrous histiocytoma: a review article. The American Journal of Surgical Pathology 6: 761-771

12. Nielsen S W 1952 Extraskeletal giant cell tumor in a cat. Cornell Veterinarian 42: 304-311

13. Pool R R 1990 Tumors and tumorlike lesions of joints and adjacent soft tissues. In Moulton J E (ed.) Tumors in domestic animals (3rd edn). University of California, Berkeley: 102-156

14. Render J A, Harrington D D, Wells R E, Dunstan R W, Turek J J, Boosinger T R 1983 Giant cell tumor of soft parts in six horses. Journal of the American Veterinary Medical Association 183: 790-793

15. Renlund R C, Pritzker KP H 1984 Malignant fibrous histiocytoma involving the digit in a cat. Veterinary Pathology 21: 442-444

16. Ruffolo P R, Kirkman H 1965 Malignant, transplantable, giant cell tumors of peri-articular connective tissues in Syrian golden hamsters (Mesocricetus auratus). British Journal of Cancer 19: 573-580

17. Seiler R J, Wilkinson G T 1980 Malignant fibrous histiocytoma involving the ilium in a cat. Veterinary Pathology 17: 513-517

18. Thrall D E 1994 Textbook of veterinary diagnostic radiology (2nd edn). W B Saunders, Philadelphia

19. Whitelock R G, Dyce J, Houlton J E F, Jefferies A R 1997 A Review of 30 tumours affecting joints. Veterinary Comparative Orthopaedics and Traumatology 10: 146-152 